\title{
Discrete Superior Hyperbolicity in Chaotic Maps
}

\author{
Ashish ${ }^{(D *, 1}$, Jinde Cao ${ }^{(D)} \beta, 2$, Fawaz Alsaadi ${ }^{\mathbb{D}} \ddagger, 3$ and A. K. Malik ${ }^{\mathbb{D}} \S \S, 4$ \\ *Department of Mathematics, Government College Satnali, Mahendergarh-123024, India, ${ }^{\beta}$ School of Mathematics, Southeast University, Nanjing-210096,

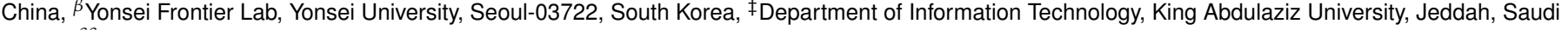 \\ Arabia, $\S_{\mathrm{B}} \mathrm{B}$. K. Birla Institute of Engineering and Technology, Pilani-333031, India.
}

ABSTRACT In the last few decades, the dynamics of one-dimensional chaotic maps have gained the tremendous attention of scientists and scholars due to their remarkable properties such as period-doubling, chaotic evolution, Lyapunov exponent, etc. The term hyperbolicity, another important property of chaotic maps is used to examine the regular and irregular behavior of the dynamical systems. In this article, we deal with the hyperbolicity and stabilization of fixed states using a superior two-step feedback system. Due to the

KEYWORDS

Chaos Hyperbolicity Bifurcation plot Chaotic maps superiority in the chaotic evolution of one-dimensional maps in the superior system we are encouraged to examine the hyperbolicity and stabilization in chaotic maps. The hyperbolic notion, hyperbolicity in periodic states of prime order, stabilization, and the hyperbolic set of the chaotic maps are studied. The numerical, as well as experimental simulations, are carried out, followed by theorems, examples, remarks, functional plots, and bifurcation diagrams.

\section{INTRODUCTION}

Hyperbolicity, in short, is an effective and efficient tool that examines the regular and irregular behavior in nonlinear dynamical systems. In fact, it induces an invariant set for different parameter values which are responsible for the chaotic phenomena of a dynamical system. It was Poincare (1899) who first introduced the hypothesis of chaotic phenomena which is considered as an essential factor in the study of hyperbolicity and stabilization. Surprisingly, the standard chaotic map $v p(1-p)$, a model of population growth has a significant role in the simulation of hyperbolicity, invariant sets, and stability in chaos theory.

P. F. Verhlust (1845 and 1847) first established the chaotic map $v p(1-p)$ as a model of population growth, where the parameter $v$ varies in a certain range. But the dynamical appearance in any chaotic system leads to $2^{n}$ periodic cycles through a bifurcation plot. Finally, the bifurcation plot leads

\footnotetext{
Manuscript received: 13 May 2021,

Revised: 11 June 2021 ,

Accepted: 15 June 2021

1 akrmsc@gmail.com

2 jdcao@seu.edu.cn

3 fesalsaadi@kau.edu.sa

4 ajender.malik@bkbiet.ac.in (Corresponding Author)
}

to a chaotic domain which gives a set of invariant measures and is known as a hyperbolic set. Further, for a detailed study on hyperbolicity and on its stability one may refer to, Robinson (1995), Holmgren (1994), Devaney (1948), Devaney (1992), Alligood et al. (1996), Martelli (1999), Chugh et al. (2012), etc..

In the last few decades, the hyperbolicity and the stabilization in fixed and periodic states have been studied by various academicians using standard chaotic systems. In 2001, Glendinning (2001) examined the hyperbolicity in the standard chaotic system $v p(1-p)$, for the parameter range $4<v \leq 2+\sqrt{5}$ and also established a good estimation of the expansion rate on invariant sets. In 2003, Kraft (1999) studied some analytical results on the hyperbolicity of chaotic maps for $v>4$ using Schwarzian derivative and shown that it is negative except for its critical states.

Robinson (1995) and Newhouse (1981) proved the repelling hyperbolicity on invariant sets using Schwarz Lemma for complex functions. Further, Guckenheimer (1979), Melo (1993) and Misiurewicz (1976) also described some analytical results using kneading theory and Schwarzian derivative. In 2003, Aulbach et al. (2004) using elementary calculus established that the invariant set $\Lambda_{v}$ is hyperbolic for $v>4$ and also proved that for $v>4$, the 
system is chaotic with the capacity of Bernoulli shift defined on $[0,1]$.

In the twenty first century, the chaotic maps have played a crucial role in every branch of science such as in traffic control system (Ashish et al. 2018, 2019b), cryptography, (Wang 2017; Akgul 2013), secure communication (Baptista et al. 1998), etc. Further, for a detailed study one may refer to Adiyaman (2020), Andrecut (1998), Ausloos et al. (2006), Jonassen (2002), Saha (2009), Saha (2010), Sharkovsky et al. (1993), Kumar (2020), Volos (2018), etc.

Recently, in 2021, Ashish et al. (2021) introduced a modulated logistic system and reported superior chaos through period-doubling, period-three orbit, and Lyapunov exponent. Also, they examined the elementary properties for chaotic maps in Ashish et al. (2019a), controlling chaos with applications in the traffic control system in Ashish et al. (2019b) and irregularity in Ashish et al. (2018) using twostep feedback approach.

The article is arranged into five major sections. Section 1 accommodates essential literature review and Section 2 consists of elementary results. The results on hyperbolicity and stability of periodic states of prime order are described in Section 3. An experimental simulation for hyperbolic sets in the two-step superior system is described in Section 4. Finally, all the results are summarized in Section 5.

\section{PRELIMINARIES}

This section deals with some basic entities in chaos theory that are used in further sections to determine the hyperbolicity of the chaotic maps in a superior two-step feedback system.

Definition 1. (Hyperbolicity). Let $\tilde{p}$ be a periodic point of order $n \in N$, then $\tilde{p}$ is said to be hyperbolic for the map $f$ if it satisfy $\left|\left(f^{(n)}\right)^{\prime}(\tilde{p})\right| \neq 1$ (Devaney 1948).

Definition 2. (Periodic state). Let $\tilde{p} \in X$ be a point and $f$ be a map defined on $X$. Then, $\tilde{p}$ is periodic of prime order $n$ if $f^{(n)}(\tilde{p})=\tilde{p}$ but $f^{(m)}(\tilde{p}) \neq \tilde{p}$ for $1 \leq m<n$ (Devaney 1992).

Definition 3. (Sink and stretch states). A point $\tilde{p} \in X$ for a map $f$ is said to be sink if $\left|f^{\prime}(p)\right|<1$ and is said to be stretch if $\left|f^{\prime}(p)\right|>1$ (Devaney 1992).

Definition 4. (Superior two-step feedback system). For an initiator $p \in X$, the iterative sequence $\left\{p_{n}\right\}$ defined by $p_{n+1}=p_{n}-\alpha_{n}\left(p_{n}-f\left(p_{n}\right)\right)$, where $0 \leq \alpha_{n} \leq 1$ is said to be superior iterative orbit and the complete process is known as superior two-step feedback system (Mann 1953).

Definition 5. (Hyperbolic set). Let $\Lambda$ be an invariant set for the map $f$ defined on $X$, that is, $f(\Lambda)=\Lambda$. Then, the invariant set $\Lambda$ is said to be hyperbolic, if it satisfy $\left|\left(f^{(n)}\right)^{\prime}(p)\right| \geq K \theta^{n}$, for $p \in \Lambda, n \geq 1, \theta>1$ and constant $K>0$ (Devaney 1948).
HYPERBOLICITY AND STABILIZATION ANALYSIS

Throughout this section, we deal with the analytical as well as numerical simulations for hyperbolicity and stabilization of fixed and periodic states of chaotic maps in a superior two-step feedback system. The hyperbolicity and stabilization in fixed and periodic states is described, followed by some theorems, examples and remarks. Therefore, let us consider $f_{\mu}$ be a chaotic map defined on $X$. Then, from Definition 4 , for the superior two-step feedback system, we can write

$$
p_{n+1}=p_{n}-\alpha\left(p_{n}-f_{v}\left(p_{n}\right)\right)=S_{\alpha, v}(p) . \quad \text { (say) }
$$

Then, for an initiator $p_{0} \in X$ and using (1) we obtain the following iterative sequence,

$$
S O^{+}\left(p_{0}\right)=\left\{p_{0}, p_{1}, p_{2}, \ldots\right\}
$$

and is said to be forward iterative sequence for an initiator $p_{0}$. Similarly, we get the relation

$$
S O^{-}\left(p_{0}\right)=\left\{p_{0}, p_{-1}, p_{-2}, \ldots\right\}
$$

and is said to be backward iterative sequence for an initiator $p_{0}$. Then, from (2) and (3), we obtain the following complete iterative sequence

$$
\begin{aligned}
S O\left(p_{0}\right) & =\left\{p_{0}, p_{-1}, p_{-2}, \ldots\right\} \bigcup\left\{p_{0}, p_{1}, p_{2}, \ldots\right\}, \\
& =\left\{\ldots, p_{-2}, p_{-1}, p_{0}, p_{1}, p_{2}, \ldots\right\}, \\
& =\left\{p_{n}: n \in Z\right\} .
\end{aligned}
$$

Also, for the $n^{\text {th }}$ iterate of the chaotic map $f_{v}$ using (1) we obtain

$$
p_{n+1}=(1-\alpha) p_{n}+\alpha f\left(f_{v}^{n-1}\left(p_{0}\right)\right)_{n \in N}=S_{\alpha, v}^{(n)}(p) .
$$

Thus, it is noticed that in a casual dynamical system the forward iterative sequence (2) is named as the superior orbit for an initiator $p_{0} \in X$. Therefore, using the relation (4), first we introduce the definition of hyperbolicity followed by a few examples and then prove the stability results using a superior two-step feedback system.

\section{Hyperbolicity}

Hyperbolicity, another eminent property of chaotic maps is illustrated to examine the regular and irregular movements in nonlinear systems. Therefore, this subsection deals with the hyperbolicity in fixed and periodic states using a superior two-step feedback system.

Definition 6. Let $S_{\alpha, v}(p)$ be the superior two-step system and $f_{v}$ be a chaotic map defined on $X$. Then, the point $\tilde{p} \in X$ of prime order $n$ is said to be superior hyperbolic of order- $n$ if it satisfy $\left|\left(S_{\alpha, v}^{(n)}\right)^{\prime}(\tilde{p})\right| \neq 1$, where $\alpha \in(0,1), n \in N$ and $v>0$. 
Example 1. Let us consider $S_{\alpha, v}(p)=p-\alpha\left(p-f_{v}(p)\right)$ be the superior two-step system and $f_{v}(p)=v p(1-p)$ be the chaotic map, where $v \in[0,4.22]$. Then, determine the domain of parameter $v$ for which the fixed-point $\tilde{p} \in[0,1]$ admits hyperbolicity.

Solution. Since $S_{\alpha, v}(p)=p-\alpha\left(p-f_{v}(p)\right)$ and $f_{v}(p)=$ $v p(1-p)$, where $v \in[0,4.22]$. Then, from Definition 1 for superior hyperbolicity, we can say

$$
\begin{aligned}
\left|\left(S_{\alpha, v}\right)^{\prime}(p)\right| & =\left|1-\alpha+\alpha f_{v}^{\prime}(p)\right|, \\
& =|1-\alpha+\alpha(v-2 v p)| .
\end{aligned}
$$

Also, the point $\tilde{p}=0$ and $\tilde{p}=1-\frac{1}{v}$ are the two fixed points of prime order one of the system $S_{\alpha, v}(p)$. Therefore, substituting $\tilde{p}$ one by one in (5), we obtain

$$
\left|\left(S_{\alpha, v}\right)^{\prime}(0)\right|=|1-\alpha+\alpha v|
$$

and

$$
\begin{aligned}
\left|\left(S_{\alpha, v}\right)^{\prime}\left(1-\frac{1}{v}\right)\right| & =\mid 1-\alpha+\alpha\left(v-2 v\left(1-\frac{1}{v}\right) \mid,\right. \\
& =|1-\alpha+\alpha(2+v-2 v)|, \\
& =\mid 1+\alpha-\alpha v) \mid .
\end{aligned}
$$

Since the growth-rate parameter $v \in[0,4.22]$ and $\alpha \in$ $(0,1)$, therefore, it is clear from (6) and (7) that both the fixed states are hyperbolic when $v \neq 1$, that is, $v \in$ $(0,1) \cup(1,4.22]$. Figure 1 , shows the hyperbolic behavior of the fixed states at $v=1$. To understand more about the hyperbolicity of fixed and periodic states, the graphical plot of the trajectories for the functions $S_{\alpha, v}(p)$ and $S_{\alpha, v}^{2}(p)$ is drawn in Figures 1-4. It is interesting to see that all the fixed and periodic states shown in Figures 1-4 are satisfied by Definition 1 of hyperbolicity. Figure 1 shows the functional plot using the superior system $S_{\alpha, v}(p)$ for $v=1, v>1$ and $v<1$. For $v=1$, the diagram shows that the fixed point 0 is completely hyperbolic, that is, $\left|\left(S_{\alpha, 1}\right)^{\prime}(0)\right| \neq 1$. While the bifurcation plot in Figure 2 shows that at $v=1$ the trajectory approaches to the fixed point $1-\frac{1}{v}$ and then again at $v=3.2$ the fixed point $1-\frac{1}{v}$ bifurcates into the hyperbolic periodic point of order 2. Further, Figure 3 shows the hyperbolicity of periodic points of order 2 for the system $S_{\alpha, v}^{2}(p)$. For $v=3.2$, $v<3.2$ and $v>3.2$ it admits completely hyperbolic state, hyperbolic repelling state and hyperbolic attracting state, respectively. Moreover, the magnified Figure 4 represents the hyperbolicity in higher order periodic points.

Example 2. Let us consider $S_{\alpha, v}(p)=p-\alpha\left(p-f_{v}(p)\right)$ be the superior two-step system and $f_{v}(p)=v p(1-p)^{m}$, where $m>1$ and $v>0$ be a chaotic map. Then, determine the domain of parameter $v$ for which the fixed point $\tilde{p} \in[0,1]$ admits hyperbolicity.

Solution. Since $S_{\alpha, v}(p)=p-\alpha\left(p-f_{v}(p)\right)$ and $f_{v}(p)=$ $v p(1-p)^{m}$. Then, from the above definition of hyperbolicity, we have

$$
\begin{aligned}
\left|\left(S_{\alpha, v}\right)^{\prime}(p)\right| & =\left|1-\alpha+\alpha f_{v}^{\prime}(p)\right|, \\
& =\left|1-\alpha+\alpha\left(v(1-p)^{m}-v p m(1-p)^{m-1}\right)\right| .
\end{aligned}
$$

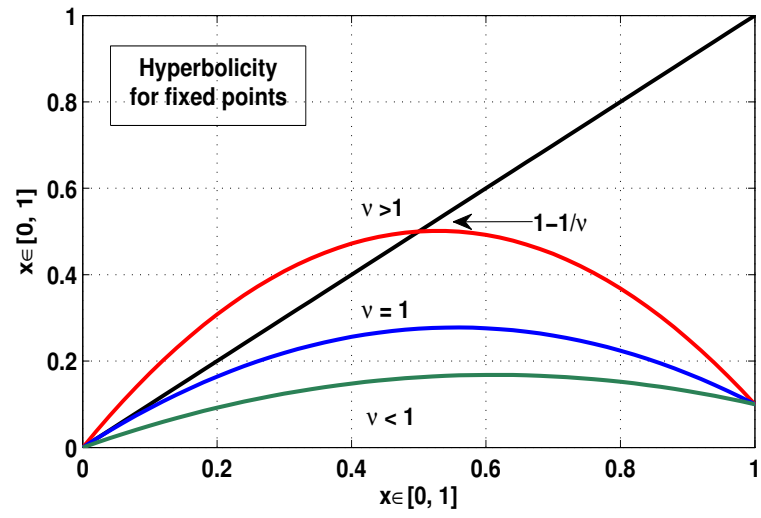

Figure 1 Functional plot for $v p(1-p)$ in $S_{\alpha, v}(p)$ for $v>1, v<1$ and $v=1$

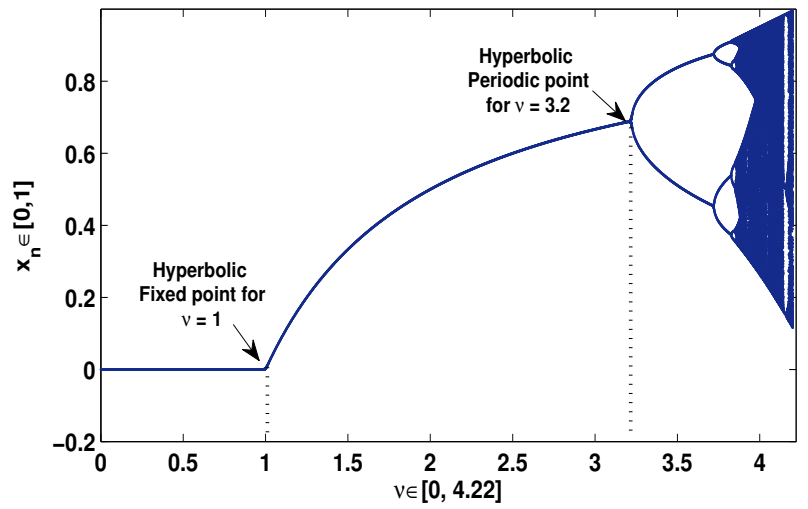

Figure 2 Bifurcation plot for $v p(1-p)$ in $S_{\alpha, v}(p)$ for $0 \leq v \leq 4.22$

Also, the point $\tilde{p}=0$ and $\tilde{p}=1-\frac{1}{\sqrt[m]{v}}$ are the two fixed point of prime order one for the system $S_{\alpha, v}(p)$. Therefore, substituting $\tilde{p}$ one by one in (8), we obtain

$$
\begin{aligned}
\left|\left(S_{\alpha, v}\right)^{\prime}(0)\right|=|1-\alpha+\alpha v| & \\
\left|\left(S_{\alpha, v}\right)^{\prime}\left(1-\frac{1}{\sqrt[m]{v}}\right)\right| & \left.=\mid 1-\alpha+\alpha-v \alpha m\left(1-\frac{1}{\sqrt[m]{v}}\right)\left(\frac{1}{\sqrt[m]{v}}\right)^{m-1}\right) \mid \\
& \left.=\mid 1-v \alpha m\left(1-\frac{1}{\sqrt[m]{v}}\right)\left(\frac{1}{\sqrt[m]{v}}\right)^{m-1}\right) \mid \\
& =\left|1-\alpha m\left(v^{1 / m}-1\right)\right| .
\end{aligned}
$$




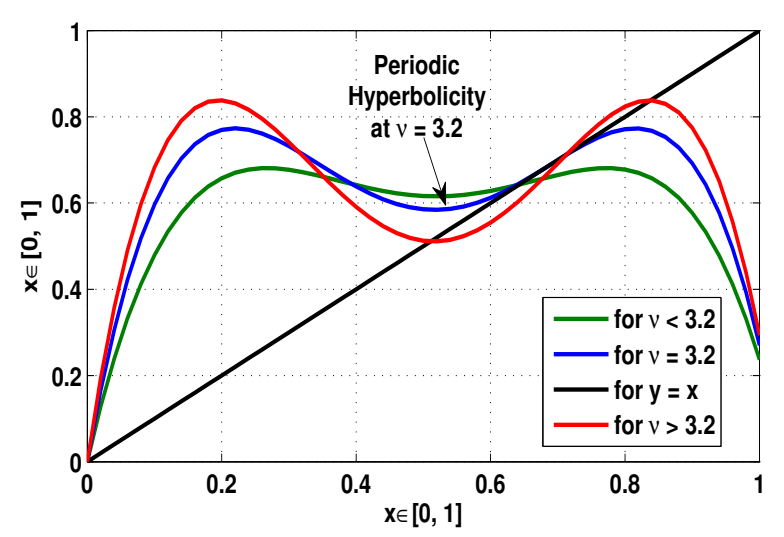

Figure 3 Periodic plot for $v p(1-p)$ in $S_{\alpha, v}^{2}(p)$ for $v=3.2, v>3.2, v<3.2$

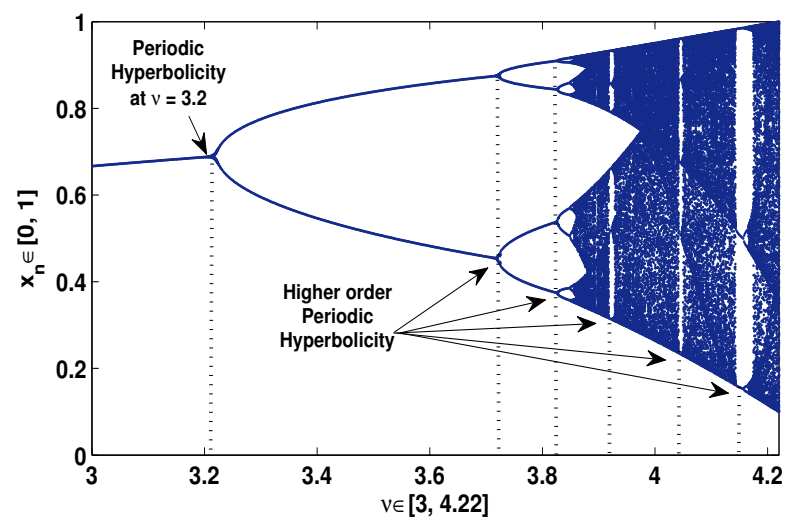

Figure 4 Hyperbolic periodic plot for $v p(1-p)$ in $S_{\alpha, v}(p)$ for $3 \leq v \leq 4.22$

Since the growth-rate parameter $v \in\left(0, v_{\max }\right]$ and $\alpha \in(0,1)$, thus, it is clear from (8) and (9) that both the fixed point are hyperbolic when $v \neq 1$, that is, $v \in(0,1) \cup\left(1, v_{\max }\right]$. In particular, for $m=2$ and $v \in$ $(0,1) \cup(1,7.2]$, Figure 5 and 6 , shows the hyperbolic fixed and periodic states. Figure 5 represents the functional plot for the quadratic map $v p(1-p)^{2}$ using superior system $S_{\alpha, v}(p)$ for $v=1, v>1$ and $v<1$. For $v=1$ it shows that the fixed state 0 is completely hyperbolic, that is, $\left|\left(S_{\alpha, 1}\right)^{\prime}(0)\right| \neq 1$ for each $\alpha \in(0,1)$. While the Figure 6 shows that the at the hyperbolic state $v=1$ the trajectory approaches to the fixed state $1-\frac{1}{\sqrt[2]{v}}$ and then again at $v=4.4$ the fixed state $1-\frac{1}{\sqrt[2]{v}}$ bifurcates into the hyperbolic periodic fixed states of order 2 . Further, as $v$ approaches through 4.4 the hyperbolic states for higher order periodic states also exists as shown in Figure 6.

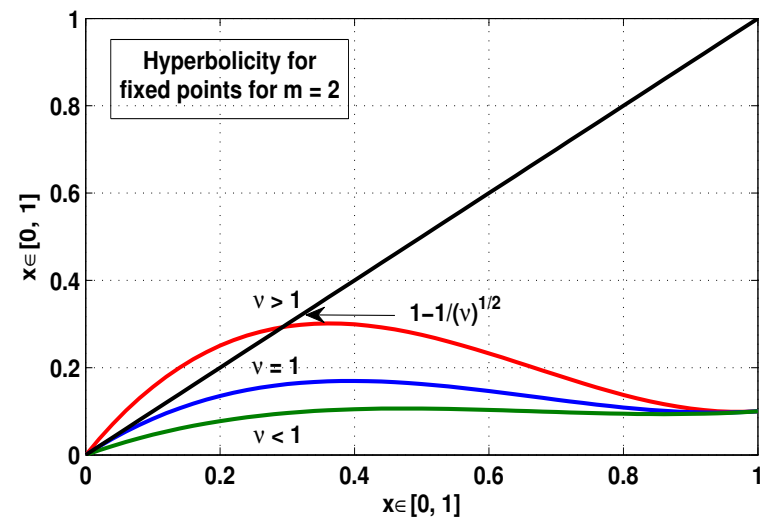

Figure 5 Functional plot for $v p(1-p)^{2}$ in $S_{\alpha, v}(p)$ for $v>1, v<1$ and $v=1$

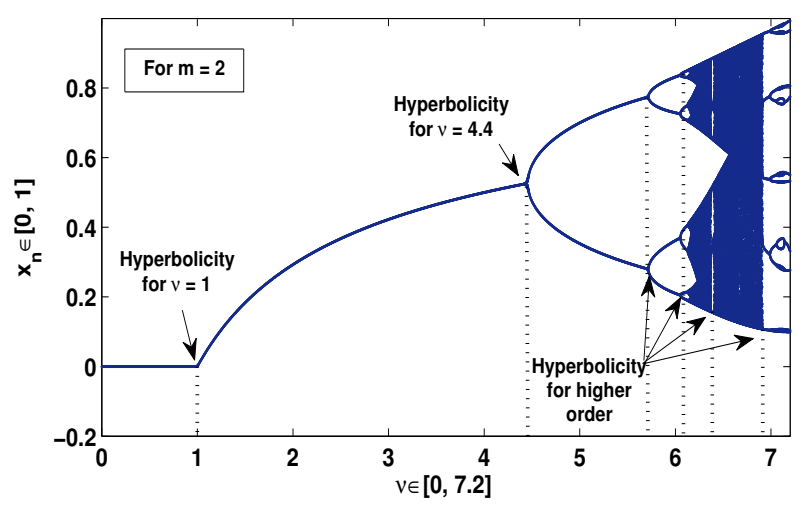

Figure 6 Hyperbolic periodic plot for $v p(1-p)^{2}$ in $S_{\alpha, v}(p)$ for $0<v \leq 7.2$

\section{Stabilization}

In this subsection, we deal with the stabilization of hyperbolic fixed and periodic states for chaotic maps using a superior two-step feedback system. The main results are followed by corollaries and remarks:

Theorem 1. Let $S_{\alpha, v}(p)$ be a superior recursive system and $f_{v}$ be a chaotic map defined on X. Also, let $\tilde{p}$ be a hyperbolic fixed state for $f_{v}$ such that $\left|\left(S_{\alpha, v}\right)^{\prime}(\tilde{p})\right|<1$. Then, for $\tilde{p} \in X$, there exists a neighbourhood $Y$ such that for each $p \in Y$, we obtain

$$
\begin{array}{ll} 
& S_{\alpha, v}^{n}(p) \rightarrow \tilde{p} \text { as } n \rightarrow \infty, \\
\text { or } \quad & \lim _{n \rightarrow \infty} S_{\alpha, v}^{n}(p)=\tilde{p} .
\end{array}
$$

Proof. Let $f_{v}$ be a chaotic map defined on $X$ with a hyperbolic fixed state $\tilde{p} \in X$. Then, there exists a number $\kappa>0$, however small, such that

$$
\begin{aligned}
& \left|\left(S_{\alpha, v}\right)^{\prime}(p)\right|<1, \quad \text { for } \quad p \in[\tilde{p}-\kappa, \tilde{p}+\kappa], \text { that is, } \\
& \left|\left(S_{\alpha, v}\right)^{\prime}(p)\right|<P<1, \quad \text { for } \quad p \in[\tilde{p}-\kappa, \tilde{p}+\kappa] .
\end{aligned}
$$

Then, from the statement of Mean Value Theorem and 
using Definition 2 of periodic state, we can write

$$
\begin{aligned}
\left|S_{\alpha, v}(p)-\tilde{p}\right| & =\left|S_{\alpha, v}(p)-S_{\alpha, v}(\tilde{p})\right|, \\
& =\left|\left(S_{\alpha, \mu}\right)^{\prime}(s)\right||p-\tilde{p}|, \quad s \in[p, \tilde{p}] \\
& <P|p-\tilde{p}|, \\
& \leq|p-\tilde{p}|, \quad(\because P<1) \\
& \leq \kappa, \quad(\because|p-\tilde{p}|<\kappa)
\end{aligned}
$$

that is, $\left|S_{\alpha, v}(p)-\tilde{p}\right| \leq \kappa$.

Thus, $S_{\alpha, v}(p) \in[\tilde{p}-\kappa, \tilde{p}+\kappa]$, for each $p \in[\tilde{p}-\kappa, \tilde{p}+\kappa]$. Inductively, it is also clear that for each $p \in[\tilde{p}-\kappa, \tilde{p}+\kappa]$, we can say

$$
S_{\alpha, v}^{n}(p) \in[\tilde{p}-\kappa, \tilde{p}+\kappa], \quad \text { for each } n \in N .
$$

Then, again using the statement of Mean Value Theorem for the $n^{\text {th }}$ iterate of the system $S_{\alpha, v}(p)$, we obtain

$$
\begin{aligned}
\left|S_{\alpha, v}^{n}(p)-\tilde{p}\right| & =\left|S_{\alpha, v}^{n}(p)-S_{\alpha, v}^{n}(\tilde{p})\right|, \\
& =\left|\left(S_{\alpha, v}^{(n)}\right)^{\prime}(s)\right||p-\tilde{p}|, \quad \text { for } s \in[p, \tilde{p}] .
\end{aligned}
$$

Now, from Devaney's (Devaney 1992) Definition for Chain rule of product along a cycle, we can write

$$
\left|\left(S_{\alpha, v}^{(n)}\right)^{\prime}(p)\right|=\prod_{i=0}^{n-1} S_{\alpha, v}^{\prime}\left(S_{\alpha, v}^{i}(p)\right),
$$

for $p \in[\tilde{p}-\kappa, \tilde{p}+\kappa]$ and $n \in N$. Then, from (10) and (14), we get

$$
\begin{aligned}
& \left|\left(S_{\alpha, v}^{(n)}\right)^{\prime}(p)\right|=\prod_{i=0}^{n-1} S_{\alpha, v}^{\prime}\left(S_{\alpha, v}^{i}(p)\right)<1, \\
& \text { that is, } \quad\left|\left(S_{\alpha, v}^{(n)}\right)^{\prime}(p)\right|<P^{n}<1 .
\end{aligned}
$$

Then, from the relation (13) and (15), we find

$$
\left|S_{\alpha, v}^{n}(p)-\tilde{p}\right|<P^{n}|p-\tilde{p}|<\kappa .
$$

Thus, $S_{\alpha, v}^{n}(p) \in[\tilde{p}-\kappa, \tilde{p}+\kappa]$, for each $p \in[\tilde{p}-\kappa, \tilde{p}+\kappa]$. Hence taking $n \rightarrow \infty$ in (16), we get the required result

$$
\left|S_{\alpha, v}^{n}(p)-\tilde{p}\right|<P^{n}|p-\tilde{p}| \rightarrow 0,
$$

that is, $\lim _{n \rightarrow \infty} S_{\alpha, v}^{n}(p)=\tilde{p}$.

Hence proved.

Theorem 2. Let $S_{\alpha, v}(p)$ be a superior recursive system and $f_{v}$ be a chaotic map defined on X. Let $\tilde{p}$ be a hyperbolic state of order $n$ satisfying $\left|\left(S_{\alpha, v}^{(n)}\right)^{\prime}(\tilde{p})\right|<1$. Then, for $\tilde{p} \in X$, there exists a neighbourhood $Y$ such that for each $p \in Y$, we have

$$
\begin{aligned}
& \quad S_{\alpha, v}^{(n k)}(p) \rightarrow \tilde{p} \text { as } k \rightarrow \infty . \\
& \text { or } \quad \lim _{k \rightarrow \infty} S_{\alpha, v}^{(n k)}(p)=\tilde{p} .
\end{aligned}
$$

Proof. Since $S_{\alpha, v}(p)$ is a superior system and $\tilde{p}$ is a periodic state of $f_{v}$, then, there exists a number $\kappa>0$, however small, such that

$$
\begin{aligned}
& \left|\left(S_{\alpha, v}^{(n)}\right)^{\prime}(p)\right|<1, \quad \text { for } \quad p \in[\tilde{p}-\kappa, \tilde{p}+\kappa], \text { that is, } \\
& \left|\left(S_{\alpha, v}^{(n)}\right)^{\prime}(p)\right|<P^{n}<1, \quad \text { for } \quad p \in[\tilde{p}-\kappa, \tilde{p}+\kappa] .
\end{aligned}
$$

Similarly for an arbitrary $k \in N$, we can say

$$
\left|\left(S_{\alpha, v}^{(n k)}\right)^{\prime}(p)\right|<P^{(n k)}<1 .
$$

Then, using Mean Value Theorem, for the system $\left(S_{\alpha, v}^{(n k)}\right)^{\prime}(p)$, we obtain

$$
\begin{aligned}
\left|S_{\alpha, v}^{(n k)}(p)-\tilde{p}\right| & =\left|S_{\alpha, v}^{(n k)}(p)-S_{\alpha, v}^{(n k)}(\tilde{p})\right|, \\
& =\left|\left(S_{\alpha, v}^{(n k)}\right)^{\prime}(s)\right||p-\tilde{p}|, \quad \text { for } s \in[p, \tilde{p}], \\
& <P^{n k}|p-\tilde{p}|,
\end{aligned}
$$

that is, $\left|S_{\alpha, v}^{(n k)}(p)-\tilde{p}\right|<\kappa$.

Thus, $S_{\alpha, v}^{(n k)}(p) \in[\tilde{p}-\kappa, \tilde{p}+\kappa]$, for each $p \in[\tilde{p}-\kappa, \tilde{p}+\kappa]$. Hence taking as $k \rightarrow \infty$ in (19), we obtain

$$
\begin{array}{ll} 
& \left|S_{\alpha, v}^{(n k)}(p)-\tilde{p}\right|<P^{n k}|p-\tilde{p}| \rightarrow 0, \\
\text { that is, } \quad & \lim _{k \rightarrow \infty} S_{\alpha, v}^{(n k)}(p)=\tilde{p} .
\end{array}
$$

Hence proved.

Corollary 1. Let $S_{\alpha, v}(p)$ be a superior recursive system and $f_{v}$ be a chaotic map defined on X. Also, let $\tilde{p}$ be a hyperbolic state for the map $f_{v}$ such that $\left|\left(S_{\alpha, v}\right)^{\prime}(\tilde{p})\right|<1$. Then, for $\tilde{p} \in X$, there exists a neighbourhood $Y$ such that for each $p \in Y$, we have

$$
\begin{aligned}
S_{\alpha, v}^{(-n k)}(p) & \rightarrow \tilde{p} \quad \text { as } \quad k \rightarrow \infty \\
\text { or } \quad \lim _{k \rightarrow \infty} S_{\alpha, v}^{(-n k)}(p) & =\tilde{p} .
\end{aligned}
$$

Proof. The proof may be illustrated by using Theorem 1 and 2.

Remark 1. Let $S_{\alpha, v}(p)$ be the superior recursive system and $\tilde{p}$ be a hyperbolic periodic state for the map $f_{v}$ satisfying $\left|\left(S_{\alpha, v}^{(n)}\right)^{\prime}(p)\right|<1$. Then, $\tilde{p} \in X$ is said to be hyperbolic stable of order- $n$. For $0<v<1$, the fixed point $\tilde{p}=0$ is hyperbolic stable and for $0<v<3.2$, the periodic state $\tilde{p}$ is hyperbolic stable as shown in Fig. 1 and 3.

Remark 2. Let $S_{\alpha, v}(p)$ be the superior recursive system and $\tilde{p}$ be a hyperbolic periodic fixed point for the map $f_{v}$ satisfying $\left|\left(S_{\alpha, v}^{(n)}\right)^{\prime}(p)\right|>1$. Then, $\tilde{p}$ is said to be hyperbolic unstable of order- $n$. 


\section{HYPERBOLIC SET}

In the earlier sections, the hyperbolicity of fixed states and their stability is described in chaotic maps using the superior two-step system. But this section deals with the hyperbolic sets in chaotic maps using the superior two-step system. Therefore, let us start with the chaotic map $v p(1-p)$ and the system $S_{\alpha, v}(p)$. Figure 7 shows the functional plot of the system $S_{\alpha, v}(p)$ which gives a parabola and intercept at $(0,0)$ and $(1,0)$. For $p=\frac{1}{2}$ the system $S_{\alpha, v}(p)$ approaches a maximum $\frac{v}{4.22}>1$ if and only if $v>4.22$. In 1992, Devaney (1992) introduced that quadratic map $v p(1-p)$ for $v>4$ admits the following Cantor set representation

$$
\Lambda_{v}=\bigcap_{n=1}^{\infty} I_{i_{0} i_{1} \ldots i_{n}}
$$

where $I_{i_{0}} \supset I_{i_{0} i_{1}} \supset \ldots \supset I_{i_{0} i_{1} \ldots i_{n}}$ is a nested sequence of closed intervals. Afterward, Kraft (1999) and Aulbach et al. (2004) also examined that for $v>4$, the set $\Lambda_{v}$ is hyperbolic, since it satisfies $\left|f_{v}^{\prime}(p)\right|>1$ for $v>4$. Therefore, looking into the potential of superior system in dynamical systems, the future work of hyperbolic set is studied in this section.

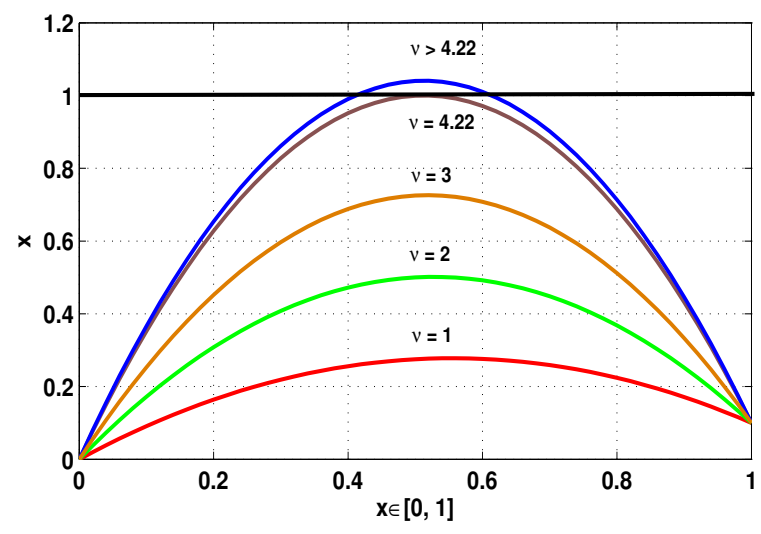

Figure 7 Functional plot for the system $S_{\alpha, v}(p)$ for $0<v \leq 4.22$ and $v>4.22$

Now, to examine hyperbolicity in $S_{\alpha, v}(p)$, let us take the quadratic map $v p(1-p)$, where $v>4.22$. Figure 7 shows the functional plot for the system $S_{\alpha, v}(p)$ for $v=1,2,3,4.22$ and 4.5 in different color radiations. It is observed that as the value of the growth-rate parameter $v$ lies in the closed interval $(0,4.22]$ the parameter $p \in[0,1]$. But as the value of $v$ approaches through 4.22, the functional plot also approaches beyond the closed interval $[0,1]$. That means, a Cantor set representation $\Lambda_{\alpha, v}$ admits a nested sequence of closed intervals which is hyperbolic for $v>4.22$. Moreover, it is examined that at $v=4.22$ all the higher order iterations of the system $S_{\alpha, v}(p)$ lies in $[0,1]$ as shown in Figure 8 for $S_{\alpha, v}(p), S_{\alpha, v}^{2}(p)$ and $S_{\alpha, v}^{3}(p)$. But as $v$ approaches beyond 4.22 , all the higher order iterations goes to $\pm \infty$ as shown in Figure 9. Also, from the Figure 10 it is analyzed that the bifurcation characteristic stops when $v=4.22$ and the hyperbolic set $\Lambda_{\alpha, v}$ exists for $v>4.22$.

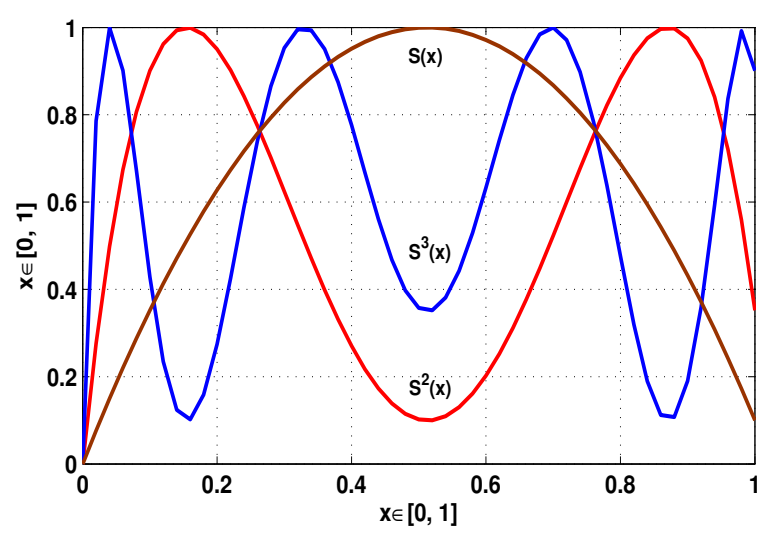

Figure 8 Functional plot $S_{\alpha, v}(p), S_{\alpha, v}^{2}(p)$ and $S_{\alpha, v}^{3}(p)$ for $v=4.22$

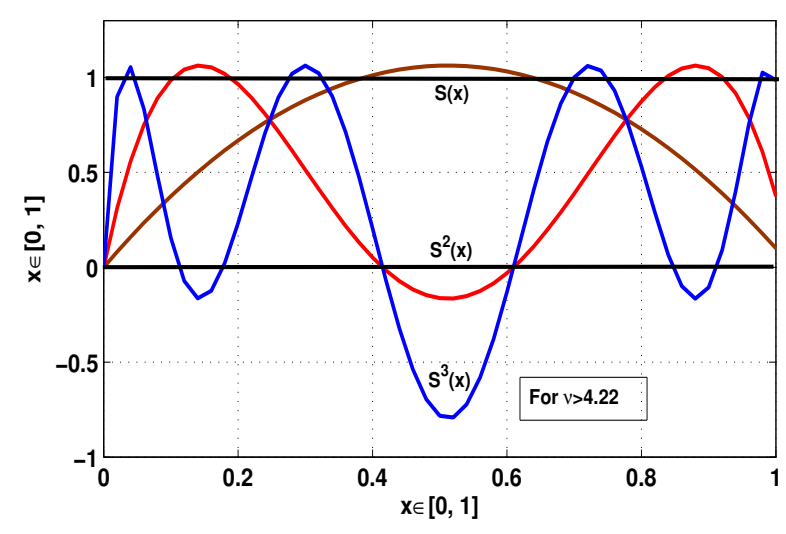

Figure 9 Functional plot $S_{\alpha, v}(p), S_{\alpha, v}^{2}(p)$ and $S_{\alpha, v}^{3}(p)$ for $v>4.22$

Remark 3. From the above analysis it is noticed that as $v \in$ $[0,4.22], S_{\alpha, v}^{n}(p) \subset[0,1]$ and for $v>4.22, S_{\alpha, v}^{n}(p) \supset[0,1]$. For a particular value $v=4.5, S_{\alpha, v}^{n}(p) \rightarrow \infty$ as $n \rightarrow \infty$.

Remark 4. For $v>4.22$ the quadratic map $v p(1-p)$ in $S_{\alpha, v}(p)$ admits a compact invariant set $\Lambda_{\alpha, v}$ for $v>4.22$, which is hyperbolic for the system $S_{\alpha, v}(p)$. 


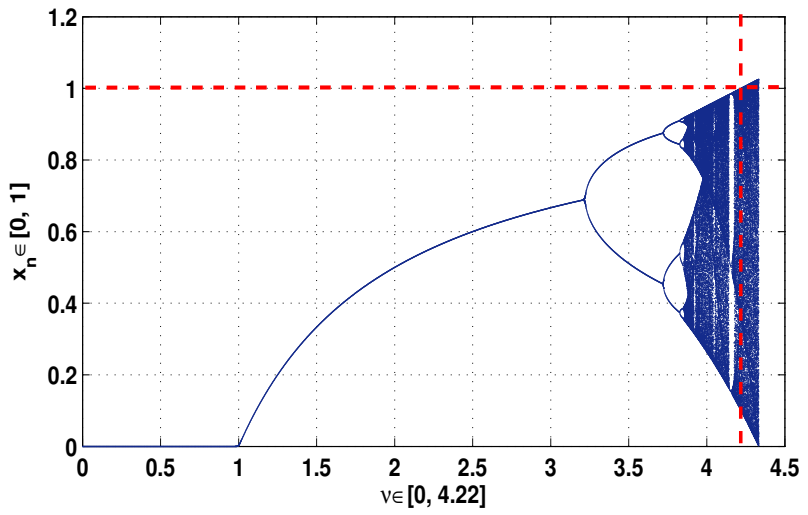

Figure 10 Bifurcation plot for the system $S_{\alpha, v}(p)$ for $0 \leq v \leq 4.5$

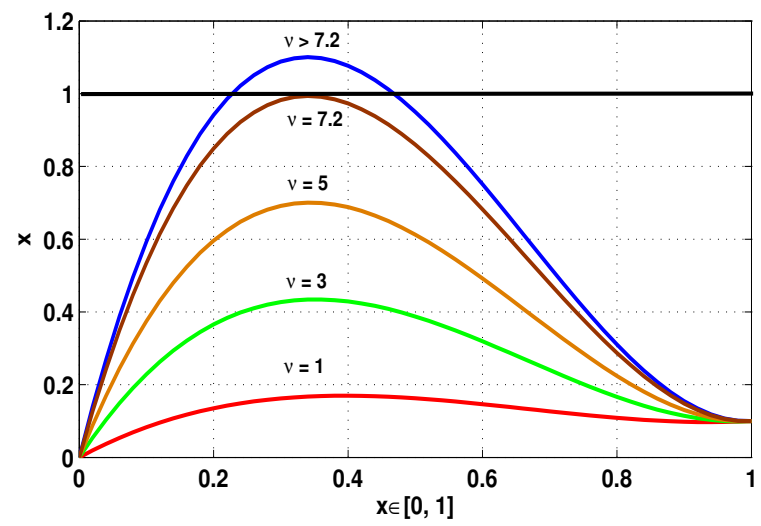

Figure 11 Functional plot for the system $S_{\alpha, v}(p)$ for $v \leq 7.2$ and $v>7.2$

Similarly, we consider an another cubic map $f_{v}(p)=$ $v p(1-p)^{2}$, where $v \in[0,7.2]$ and $p \in[0,1]$. Figure 11 shows the functional plot for the different parameter values of $v$. Taking $v=1,3,5$ and 7.2 the orbit of iteration $p_{n} \in[0,1]$. But as the value of parameter $v$ is approached through 7.2 the functional plot of the map approaches outside the closed interval $[0,1]$ as shown in Figure 11. Further, the Figure 12 shows that the functional plot of the higher order iterations such as $S^{3}(p), S^{2}(p)$ and $S(p)$ also lies in $[0,1]$ for each $v \in[0,7.2]$. But as $v$ approaches beyond 7.2 the functional plot of higher order tends to $\pm \infty$ as $n \rightarrow \infty$ as shown in Figure 13. Moreover, from the bifurcation plot, Figure 14 it is clear that for $v>7.2$ we obtain a compact invariant set $\Lambda_{\alpha, v}$ in which the function iteration approaches beyond the closed interval $[0,1]$, that is, $S_{\alpha, v}^{n}([0,1]) \supset[0,1]$. Hence $\Lambda_{\alpha, v}$ is hyperbolic set for $v>7.2$.

Remark 5. It is observed that for $v \in[0,7.2]$ the functional iteration $S_{\alpha, v}^{n}([0,1]) \subset[0,1]$ and for $v>7.2, S_{\alpha, v}^{n}([0,1]) \supset$ $[0,1]$. Further, for $v>7.2$, it is also determined that the interval of recursive sequence $S_{\alpha, v}^{n}(p)$ is not same as the interval of an initiator $p \in[0,1]$ as shown in Figures 11-14.

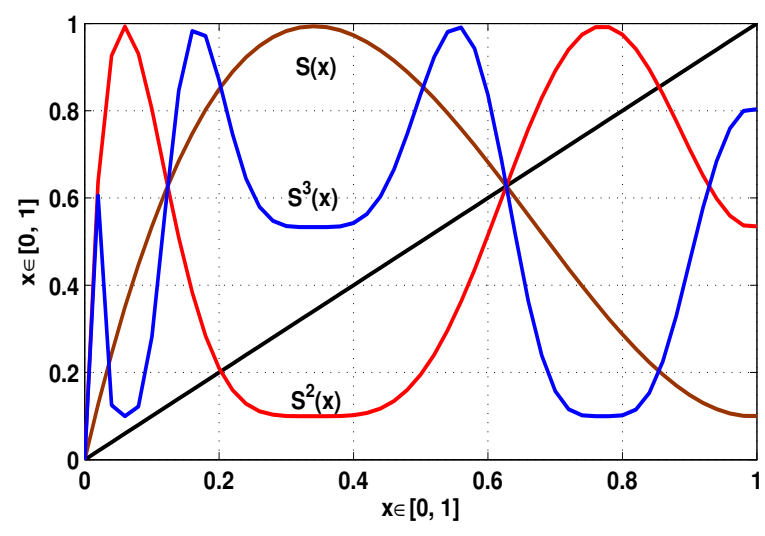

Figure 12 Functional plot $S_{\alpha, v}(p), S_{\alpha, v}^{2}(p)$ and $S_{\alpha, v}^{3}(p)$ for $v=7.2$

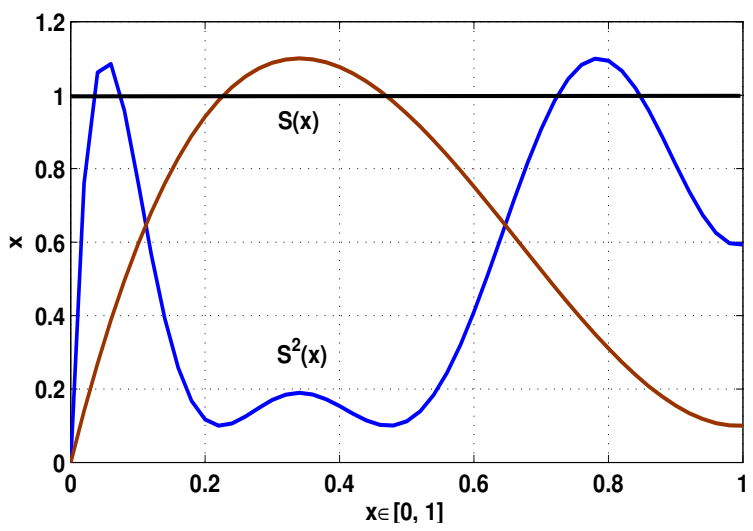

Figure 13 Functional plot $S_{\alpha, v}(p)$ and $S_{\alpha, v}^{2}(p)$ for $v=7.2$

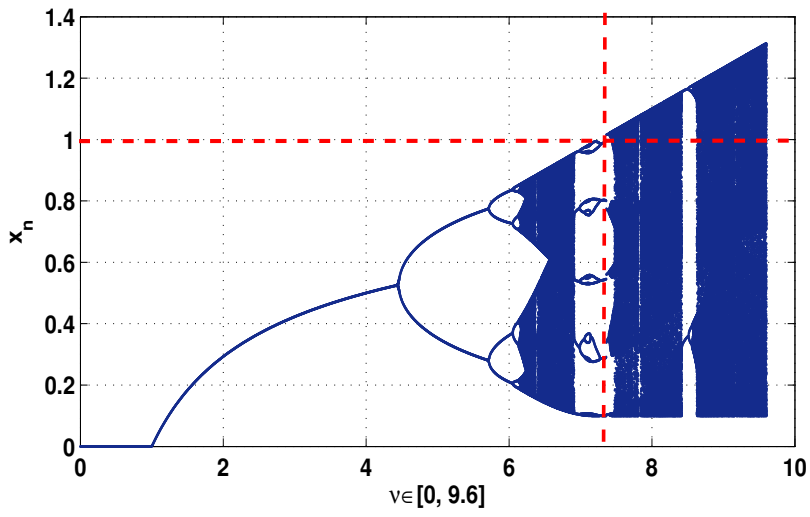

Figure 14 Bifurcation plot for the system $S_{\alpha, v}(p)$ for $0 \leq v \leq 10$ 


\section{CONCLUSION}

In this article, a two-step superior feedback approach is established to examine the hyperbolicity and the stabilization for one-dimensional chaotic maps. Throughout the study, a few mathematical results are derived and experimental simulations are carried out. Thus, we conclude the following results:

- In Section 3 the superior hyperbolic notions for the chaotic maps are derived using superior system. Definition 1, for the hyperbolic fixed and periodic states is introduced followed by the Example 1 and 2 for the chaotic maps. Further, the numerical simulations are also presented in each case.

- The hyperbolic control results for fixed and periodic state are described. Theorem 1, presents the stability in hyperbolic fixed states and Theorem 2 determines the stability in periodic states.

- In Section 4, the property of hyperbolic set is described using experimental analysis of the quadratic and cubic type maps in superior system. Moreover, it is studied that for the chaotic map $v p(1-p)$ the invariant sets $\Lambda_{\alpha, v}$ is hyperbolic for $v>4.22$ and for the cubic map $v p(1-p)^{2}$ is hyperbolic for $v>7.2$.

Further, it is emphasized that the hyperbolic property in superior system may lead to a strong interest in nonlinear systems. In the next article, we will present some applications on hyperbolicity.

\section{Acknowledgments}

This work was jointly supported by Deanship of Scientific Research (DSR) at King Abdulaziz University, Jeddah, Saudi Arabia under Grant no. FP-108-42.

\section{Conflicts of interest}

The authors declare that there is no conflict of interest regarding the publication of this paper.

\section{LITERATURE CITED}

Adiyaman, Y., S. Emiroglu, M. Ucar and M. Yildiz, 2020 Dynamical analysis, electronic circuit design and control application of a different chaotic system, Chaos Theory and Applications 02: 10-16.

Akgul, A., Kaçar, S., Aricioğlu, B., and Pehlivan, I., Text encryption by using one-dimensional chaos generators and nonlinear equations. In 2013 8th International Conference on Electrical and Electronics Engineering (ELECO), IEEE 320-323.

Alligood, K. T., T. D. Sauer and J. A. Yorke, 1996 Chaos : An Introduction to Dynamical Systems, Springer Verlag, New York Inc.

Andrecut, M., 1998 Logistic map as a random number generator, International Journal of Modern Physics B 12: 101102.
Ashish, J. Cao and R. Chugh, 2018 Chaotic behavior of logistic map in superior orbit and an improved chaos-based traffic control model, Nonlinear Dynamics 94: 959-975.

Ashish and J. Cao, 2019a A novel fixed point feedback approach studying the dynamcial behaviour of standard logistic map, International Journal of Bifurcation and Chaos 29: 1950010-16, 16 pages.

Ashish, J. Cao and R. Chugh, 2019b Controlling chaos using superior feedback technique with applications in discrete traffic models, International Journal of Fuzzy System 21: 1467-1479.

Ashish, J. Cao and R. Chugh, 2021 Discrete chaotification in modulated logistic system, International Journal of Bifurcation and Chaos 31: 2150065, 14 Pages.

Aulbach, B. and B. Kieninger, 2004 An elementary proof for hyperbolicity and chaos of the logistic maps, Journal of Difference Equations and Applications 10: 1243-1250.

Ausloos, M. and M. Dirickx, 2006 The Logistic Map and the Route to Chaos : from the Beginnings to Modern Applications, Springer Verlag, New York Inc.

Baptista, M. S., 1998 Logistic map as a random number generator, Physics Letter A 240: 50-54.

Chugh, R., M. Rani and Ashish, 2012 Logistic map in Noor orbit, Chaos and Complexity Letter 6, 167-175.

Devaney, R. L., 1948 An Introduction to Chaotic Dynamical Systems, 2nd Edition (Addison-Wesley).

Devaney, R. L., 1992 A First Course in Chaotic Dynamical Systems: Theory and Experiment, (Addison-Wesley).

Glendinning, P., 2001 Hyperbolicity of the invariant set for the logistic map with $\mu>4$, Nonlinear Analysis 47: 33233332.

Guckenheimer, J., 1979 Sensitive dependence to initial conditions for one-dimensional maps, Communications in Mathematical Physics 70: 133-160.

Holmgren, R. A., 1994 A First Course in Discrete Dynamical Systems, Springer Verlag, New York Inc.

Jonassen, T. M., 2002 On the Concept of Hyperbolicity, Oslo Univ. College Report Series 21:, ISBN 82-579-4155-7.

Kraft, R. L., 1999 Chaos, cantor sets and hyperbolicity for the logistic maps, Transactions of the American Mathematical Society 106: 400-408.

Kumar, V., Khamosh and Ashish, 2020 An empirical approach to study the stability og generalized logistic map in superior orbit, Advances In Mathematics: Scientific Journal 10: 2094-2109.

Mann, W. R., 1953 Mean value methods in iteration, Proceedings of American Mathematical Society 04: 506-510.

Martelli, M., 1999 Chaos : An Introduction to Discrete Dynamical Systems and Chaos, Wiley-Interscience Publication, New York Inc.

Melo, W. de. and S. J. van Strien, 1993 One-dimensional dynamics, Springer, Berlin.

Misiurewicz, M., 1976 Absolutely continuous measures for certain maps of an interval, Publications Mathematiques de 1'Institut des Hautes Etudes Scientifiques, 261: 459-475.

Newhouse, S. J., 1981 The abundance of wild hyperbolic set and non-smooth stable sets for diffeomorphism, Pub- 
lications Mathematiques de l'Institut des Hautes Etudes Scientifiques, 53: 17-51.

Poincare, H., 1899 Les Methods Nouvells de la Mecanique Leleste, Gauthier Villars, Paris.

Robinson, C., 1995 Dynamical Systems: Stabilily, Symbolic Dynamics, and Chaos, CRC Press.

Saha, L. M., L. Bharti and R. K. Mohanty, 2010 Study of bifurcation and hyperbolicity in discrete dynamical systems, Iranian Journal of Science and Technology, 34: 1-12.

Saha, L. M., R. K. Mohanty and L. Bharti, 2009 Hyperbolicity and chaos in discrete systems, International Journal of Applied Mathematics and Mechanics, 05: 48-56.

Sharkovsky, A. N., Y. L. Maistrenko and E. Y. Romanenko, 1993 Difference Equations and Their Applications, Kluwer Academic Publisher.

Volos, C. K., Akgul, A., Pham, V. T., and Baptista, M. S., 2018 Antimonotonicity, crisis and multiple attractors in a simple memristive circuit. Journal of Circuits, Systems and Computers, 27(02): 1850026.

Wang, X., Akgul, A., Cicek, S., Pham, V. T., and Hoang, D. V., 2017 A chaotic system with two stable equilibrium points: Dynamics, circuit realization and communication application. International Journal of Bifurcation and Chaos, 27(08): 1750130.

How to cite this article: Ashish, Cao, J., Alsaadi, F., and Malik, A. K. Discrete Superior Hyperbolicity in Chaotic Maps. Chaos Theory and Applications, 3(1), 34-42, 2021. 